\title{
Evaluation of the Card Test for Diagnosis of Human Brucellosis
}

\author{
ANNE O. RUSSELL, CHARLOTTE M. PATTON,* AND ARNOLD F. KAUFMANN \\ Center for Disease Control, Bureau of Epidemiology, Bacterial Diseases Division, Atlanta, Georgia 30333
}

Received for publication 8 December 1977

\begin{abstract}
The relative efficacy of the card test in the diagnosis of human brucellosis was evaluated by comparison with four other tests: the standard tube agglutination test, centrifugation agglutination test, 2-mercaptoethanol degradation technique, and 2-mercaptoethanol centrifugation agglutination test. A total of 1,701 serum specimens from persons with various degrees of potential exposure to brucella organisms or cross-reactive antigens were used in this study. In comparison with standard tube agglutination results, the card test had a sensitivity of $95.3 \%$ and a specificity of $84.1 \%$. The card-test antigen was nonreactive with serum specimens from tularemia patients and cholera vaccinees. Our data indicated that this test measures both immunoglobulin $\mathrm{G}$ and immunoglobulin $\mathbf{M}$ agglutinins. The value of the card test in the presumptive serological diagnosis of clinical brucellosis in humans appears to be low; however, the card test may be of value in serological surveys to delineate high-risk populations.
\end{abstract}

The brucellosis card test was originally developed for rapid screening of animal sera. For cattle, this test is generally regarded as being highly specific $(7,9)$; however, some investigators have reported its sensitivity as being relatively low (12). The low $\mathrm{pH}$ of the card test was initially reported to inhibit nonspecific agglutinins while leaving specific agglutinins unaffected (14). Conflicting reports exist on the class of immunoglobulin detected in bovine sera by the card-test antigen. Some investigators found the antigen reacted only with immunoglobulin $G$ (IgG) agglutinins (4), whereas others have observed reactions with both IgG and IgM agglutinins $(6,11)$.

Use of the card test in the diagnosis of human brucellosis has not been extensively evaluated. Buchanan et al. (3) found that $92 \%$ of 38 patients tested within 6 months after onset were cardtest positive and suggested that this test could provide laboratory support for the immediate initiation of treatment for symptomatic patients. Based on studies of 236 suspected brucella infections in humans in Iran, Nicoletti and FadaiGhotbi (8) found close agreement between the card test and standard tube aggiutination (STA) test, with 55 of 56 STA-positive sera also positive by the card test. They recommended wider utilization of the card test in diagnosing acute brucella infections, but felt that further investigation of the card test was necessary before use in the diagnosis of chronic brucellosis cases.

The purpose of this study was to determine the diagnostic value of the card test in human brucellosis in comparison with four other serological tests and to assess the class of agglutinins detected by the card test. For this study we used serum from individuals with various degrees of exposure to brucella organisms.

\section{MATERIALS AND METHODS}

Study populations. A total of 1,701 serum specimens from 1,101 individuals were tested for brucella antibody (Table 1). The serum specimens were from abattoir employees, farm families, regulatory workers involved in brucellosis eradication, tularemia patients, and cholera vaccinees.

The serum specimens from abattoir employees were obtained at three different abattoirs in the course of epidemic investigations. Of the 1,387 serum specimens from abattoir wokers, 482 were from 119 individuals who had current or prior clinical brucellosis and 905 were from 714 individuals who had various degrees of potential exposure to brucella organisms but no clinical history of brucellosis.

The serum specimens from farm families and regulatory workers were obtained during an epidemic investigation in Vermont. These were from 6 brucellosis patients, 153 asymptomatic individuals residing on dairy farms with brucellosis-infected herds, 59 asymptomatic individuals residing on dairy farms with brucellosis-free herds, and 15 regulatory workers exposed to brucellosis-infected cattle but with no history of brucellosis.

Serum specimens from 10 people with tularemia were obtained from the serum bank at the Center for Disease Control (CDC). These sera had tularemia antibody titers ranging from $1: 160$ to $1: 1,280$ when tested by the Bacterial Immunology Branch, Bureau of Laboratories, CDC, using a modification of Owen's method (10). 
TABLE 1. Serum specimens tested for brucella antibody by source and prior history of clinical brucellosis

\begin{tabular}{c|c|c|c}
\hline Source & $\begin{array}{c}\text { Prior brucel- } \\
\text { losis }\end{array}$ & $\begin{array}{c}\text { No prior bru- } \\
\text { cellosis }\end{array}$ & Total \\
\hline $\begin{array}{c}\text { Abattoir em- } \\
\text { ployees }\end{array}$ & $482(119)^{a}$ & $905(714)$ & $1,387(833)$ \\
$\begin{array}{c}\text { Farm fami- } \\
\text { lies }\end{array}$ & $16(6)$ & $212(212)$ & $228(218)$ \\
$\begin{array}{c}\text { Regulatory } \\
\text { workers }\end{array}$ & 0 & $15(15)$ & $15(15)$ \\
$\begin{array}{c}\text { Tularemia } \\
\text { patients }\end{array}$ & 0 & $10(10)$ & $10(10)$ \\
$\begin{array}{c}\text { Cholera vac- } \\
\text { cinees }\end{array}$ & 0 & $61(25)$ & $61(25)$ \\
Total & $498(125)$ & $1,203(976)$ & $1,701(1,101)$ \\
\hline
\end{tabular}

${ }^{a}$ Number of serum specimens (number of people).

${ }^{b}$ Involved in brucellosis eradication activities.

Serum specimens from cholera vaccinees were obtained from the Bacterial Immunology Branch, Bureau of Laboratories, CDC. The sera from these 25 individuals included 23 prevaccination and 38 postvaccination specimens. Only two of the group had a prior history of cholera vaccination, and none had a prior history of brucellosis. Twenty of the prevaccination specimens had vibriocidal cholera titers ranging from 1:20 to 1:320. All postvaccination specimens had titers ranging from $1: 160$ to $1: 40,960$. The vibriocidal cholera titers were determined according to the method of Benenson et al. (1).

Serological tests. The STA test was performed as described in Diagnostic Procedures for Bacterial, Mycotic and Parasitic Infections (5), and the 2-mercaptoethanol (2ME) degradation technique was according to the USDA modification of Anderson's method (15). After titers were determined by these two methods, tube dilutions were centrifuged for 10 $\min$ at $600 \times g$ and the centrifugation agglutination (CA) titers were redetermined. The postcentrifugation titers of the STA and 2ME test dilutions are designated as CA and 2ME-CA titers, respectively. For purpose of analysis, STA and CA titers $\geq 1: 160$ were considered to represent prior clinical or subclinical brucella infection. A 2ME or 2ME-CA titer $\geq 1: 20$ was considered an indication of brucella infection. The presence of $2 \mathrm{ME}$-resistant brucella agglutinins is considered indicative of current or recent brucellosis, but no criteria have been developed as to what constitutes a significant titer with these agglutinins (13).

The card test (Brewer Diagnostic Kits, Hynson, Westcott, and Dunning, Inc., Baltimore, Md.) is a macroscopic agglutination test utilizing a stained, buffered, whole-cell suspension of Brucella abortus 1119-3. Equal volumes of $0.03 \mathrm{ml}$ of test serum and antigen were mixed on a card supplied with the commercial test kit. The card was rocked slowly for $4 \mathrm{~min}$ on a mechanical rocker. According to instructions included with the kit, results were read as positive or negative based on agglutination (Fig. 1).

\section{RESULTS}

Table 2 shows a comparison of card- and tube- test results for all 1,701 sera, regardless of clinical or exposure history. Specificity and sensitivity, as used in this table, refer only to correlation of the card-test results with the respective tubetest results and not to the diagnostic accuracy of either test. The sensitivity was determined by dividing the number of card-test positive reactions that were also positive in the respective tube test by the total number of positive reactions in that tube test. Specificity was determined by dividing the number of card-test negative reactions that were also tube test negative by the total number of negative reactions in that tube test. Result concordance was determined

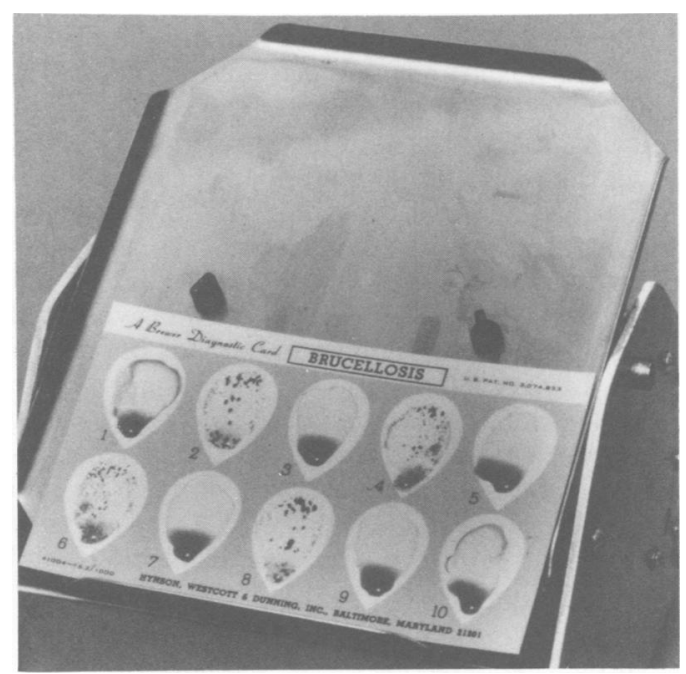

Fig. 1. Positive $(2,4,6,8)$ and negative $(1,3,5,7$ $9,10)$ brucellosis card-test reactions.

TABLE 2. Correlation of card-test with brucella tube agglutination-test titers for 1,701 human sera

\begin{tabular}{c|c|c|c|c}
\hline \multirow{2}{*}{ Results } & \multicolumn{4}{|c}{ Test procedure } \\
\cline { 2 - 5 } & STA & CA & 2ME & 2ME-CA \\
\hline $\begin{array}{c}\text { Card nega- } \\
\text { tive, tube } \\
\text { negative }\end{array}$ & 1,268 & 1,226 & 1,259 & 1,223 \\
$\begin{array}{c}\text { Card posi- } \\
\text { tive, tube } \\
\text { positive }\end{array}$ & 184 & 347 & 223 & 307 \\
$\begin{array}{c}\text { Card posi- } \\
\text { tive, tube } \\
\text { negative }\end{array}$ & 240 & 77 & 201 & 117 \\
$\begin{array}{c}\text { Card nega- } \\
\text { tive, tube } \\
\text { positive }\end{array}$ & 9 & 51 & 18 & 54 \\
$\begin{array}{c}\text { Sensitivity } \\
\text { (\%) }\end{array}$ & 95.3 & 87.2 & 92.5 & 85.0 \\
$\begin{array}{c}\text { Specificity } \\
\text { (\%) }\end{array}$ & 84.1 & 94.1 & 86.2 & 91.3 \\
\hline
\end{tabular}


by calculating the percentage of sera for which the results of both the card test and the respective tube test were in agreement. The card test correlated better with the centrifugation tests than with the noncentrifugation tests (Table 2). The best correlation was with the CA test (sensitivity, specificity, and result concordance).

Card-test results were compared with results from the four tube tests at various times after onset for 125 brucella patients (Table 3). Only results for the serum specimen collected nearest to time of clinical onset were used for each individual. All patients had clinical illness compatible with brucellosis and either a fourfold change in brucella antibody titer and/or a positive culture.

The card test had a higher number of positive reactions in all time periods than the STA, 2ME, and 2ME-CA tests (Table 3). The sensitivity of the CA and card tests was approximately equal in all time periods. The number of serum specimens having significant titers as measured by the five tests progressively declined as time after onset increased.

The specificity of the card test was evaluated by examination of sera from persons having no prior history of brucellosis. Sixty-eight serum samples from persons exposed to cross-reacting antigens were tested. None of 10 sera from tularemia patients was card-test positive or had detectable brucella antibody by the four tube tests. Of 58 sera from cholera vaccinees, 16 had

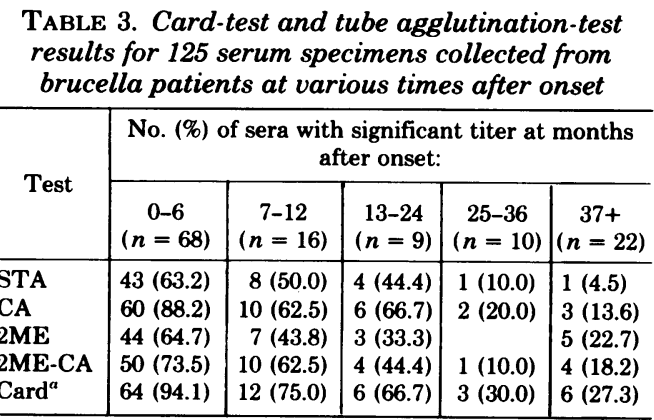

a These figures indicate the number of sera that were cardtest positive. low-level $(\leq 1: 80)$ brucella titers by the CA test, and a single serum had a 1:160 titer. None of these 58 sera was card-test positive.

Card-test positivity was also examined by prior history of clinical brucellosis and potential risk of infection in various population groups. The card-test results for a single serum specimen from each person were used in this analysis. This was either the first specimen collected or, in the case of persons with a history of clinical brucellosis, the specimen collected nearest the date of clinical onset. In all persons having a history of clinical brucellosis, the diagnosis had been confirmed by either a fourfold change in brucella antibody titer and/or a positive culture at the time of their illness.

Among high-risk abattoir workers, the card test reacted positively with serum specimens from $71.4 \%$ of current or prior brucellosis patients and $3.8 \%$ of individuals with no prior history of brucellosis (Table 4). Of 15 animal health regulatory workers and 218 farm family members, 8 persons were card-test positive. These positive persons included all 6 with current or prior clinical brucellosis and 2 of $227(0.9 \%)$ persons with no history of clinical brucellosis. The latter two individuals had had potential exposure to cattle infected with brucellosis.

\section{DISCUSSION}

The sensitivity of the card test when compared with significant titers obtained in various tube agglutination tests ranged from 85 to $95 \%$, with the highest sensitivity observed in correlation with STA results. Card-test specificity ranged from 84 to $94 \%$, with the highest specificity observed in correlation with CA results.

Previously the card-test antigen has been reported to react only with IgG antibodies which are mercaptoethanol resistant (4). If only IgG agglutinins are measured by the card-test antigen, sera with no detectable IgG $(<1: 202 \mathrm{ME}$ titers) and positive STA titers ( $\geq 1: 160)$ would be expected to give negative card-test reactions. Of 1,701 sera examined, 47 sera had both $2 \mathrm{ME}$ titers of $<1: 20$ and STA titers of $\geq 1: 160$, and 44 of these 47 sera $(93.6 \%)$ were card-test positive.

TABLE 4. Card-test positivity by prior history of clinical brucellosis in various population groups

\begin{tabular}{lccc}
\hline \multirow{2}{*}{ Group } & \multicolumn{3}{c}{ History of clinical brucellosis $^{a}$} \\
\cline { 2 - 4 } & \multicolumn{1}{c}{ Yes } & No & Total \\
\hline Abattoir workers & $85 / 119(71.4)$ & $27 / 714(3.8)$ & $112 / 833(13.4)$ \\
Animal health regulatory workers & $0 / 0$ & $1 / 15(6.7)$ & $1 / 15(6.7)$ \\
Farm families, infected herds & $6 / 6(100.0)$ & $1 / 153(0.7)$ & $7 / 159(4.4)$ \\
Farm families, noninfected herds & $0 / 0$ & $0 / 59$ & $0 / 59$ \\
Total & $91 / 125(72.8)$ & $29 / 941(3.1)$ & $120 / 1,066(11.3)$ \\
\hline
\end{tabular}

\footnotetext{
a Number of persons with positive card-test reaction per total tested (percentage).
} 
Also, sera with negative 2ME-CA and significant CA titers would be expected to give negative card-test results. Only 17 of 1,701 sera had both significant CA titers and 2ME-CA titers of $<1: 20$. Of these 17 sera, 14 (82.3\%) were card-test positive. These results and those of Patterson et al. (11) indicate that IgG is not the only class of immunoglobulin being measured by this antigen.

The card test appears to be highly sensitive in detecting recently acquired brucellosis, since $94.1 \%$ of sera collected within 6 months after onset were reactive. The sensitivity of the card test for sera collected during this time period was significantly greater than that of all the tube agglutination tests except the CA procedure (chi square: $P \leq 0.003$ ). However, its value as a presumptive diagnostic test was seriously compromised by long-term persistence of reactivity in recovered brucellosis patients and by reactivity in persons with no evidence of clinical brucellosis.

The lack of card-test reactivity in some sera having STA and CA titers $\geq 1: 160$ may be due to a blocking antibody or prozone effect resulting from the use of undiluted serum in this test. If so, this would also compromise its value as a screening procedure.

In abattoir employees, a high-risk group with frequent potential exposure to brucella organisms, 3.8\% had positive card-test reactions but no history of prior clinical brucellosis. Buchanan et al. (3) reported that $23 \%$ of 91 abattoir workers with no prior history of clinical brucellosis had a positive card-test reaction. Although these reactions frequently represent prior subclinical infection, they are false positive reactions from a diagnostic viewpoint and may lead to misdiagnosis of febrile illnesses due to other causes. In a population group such as abattoir workers with endemic brucellosis, the card test would have relatively low value as a diagnostic tool even when the annual incidence is high. For example, if the long-term annual incidence was as high as $1 \%$ in an abattoir having $1,000 \mathrm{em}$ ployees, our data suggest that $58(5.8 \%)$ of the population would have a diagnostically false positive reaction as the result of prior subclinical or clinical infection where serum specimens were obtained $>6$ months after onset. Based on our results, a positive card-test reaction in this situation would be correct in $14.7 \%(10 / 68)$ of patients with nonspecific febrile illness. The diagnostic value would be even less at lower incidence rates.

In the general population of the United States, brucellosis is a rare disease, with a current reported annual incidence of 0.07 cases per 100,000 population. The incidence of false positive card- test reactions in the general population is unknown; however, assuming a specificity as high as $99.9 \%$, the card test, with its expected incidence of 100 false positive reactions per 100,000 population, would still be of limited diagnostic value. The ratio of diagnostically correct positive reactions to false positive reactions in this situation would be 1:1,429.

Tube agglutination testing is still the method of choice for serological diagnosis of acute brucellosis. Tube testing offers the advantages of demonstrating changes in titer with paired sera and the presence or absence of 2ME-resistant antibody. The card test does appear to have potential value as a rapid, economical epidemiological tool for serological surveys to delineate high-risk populations.

\section{ACKNOWLEDGMENTS}

Appreciation is extended to John Boyce, Marshall Fox, and Dan Anderson for the collection of sera and obtaining case histories. We also thank Liz Sparger for her technical assistance.

\section{LITERATURE CITED}

1. Benenson, A. S., A. Saad, and W. H. Mosley. 1968. Serological studies in cholera. 2. The vibriocidal antibody response of cholera patients determined by a microtechnique. Bull. W.H.O. 38:277-285.

2. Buchanan, T. M., S. L. Hendricks, C. M. Patton, and R. A. Feldman. 1974. Brucellosis in the United States, 1960-1972. An abattoir-associated disease. III. Epidemiology and evidence for acquired immunity. Medicine 53:427-439.

3. Buchanan, T. M., C. R. Sulzer, M. K. Frix, and R. A. Feldman. 1974. Brucellosis in the United States, 1960-1972. An abattoir-associated disease. II. Diagnostic aspects. Medicine 53:415-425.

4. Corbel, M. J. 1972. Identification of the immunoglobulin class active in the rose bengal plate test for bovine brucellosis. J. Hyg. 70:779-795.

5. Hausler, W. J., Jr., and F. P. Koontz. 1970. Brucellosis, p. 364-378. In H. L. Bodily, E. L. Updyke, and J. O. Mason (ed.), Diagnostic procedures for bacterial, mycotic and parasitic infections, 5 th ed. American Public Health Association, New York.

6. Lambert, B., and T. E. Amerault. 1962. An evaluation of acidified plate test antigens for detecting bovine brucellosis. Am. J. Vet. Res. 23:1031-1033.

7. Nicoletti, P. 1967. Utilization of the card test in brucellosis eradication. J. Am. Vet. Med. Assoc. 151: 1778-1783.

8. Nicoletti, P., and M. M. Fadai-Ghotbi. 1971. A comparison of the tube agglutination and card tests for the diagnosis of Brucella melitensis infection in humans. Can. J. Public Health 62:443-445.

9. O'Reilly, D. J., and B. Cunningham. 1971. An assessment of the brucellosis card test. Vet. Rec. 88:590-594.

10. Owen, C. R. 1970. Francisella infections, p. 468-483. In H. L. Bodily, E. L. Updyke, and J. O. Mason (ed.), Diagnostic procedures for bacterial, mycotic and parasitic infections, 5th ed. American Public Health Association, New York.

11. Patterson, J. M., B. L. Deyoe, and S. S. Stone. 1976. Identification of immunoglobulins associated with complement fixation, agglutination, and low $\mathrm{pH}$ buffered antigen tests for brucellosis. Am. J. Vet. Res. 37: 


$$
\text { 319-324. }
$$

12. Prior, M. G., L. Niilo, and W. H. Reeker. 1975. Use of the brucellosis card test for screening cattle in Saskatchewan. Can. J. Comp. Med. 39:107-109.

13. Reddin, J. L., R. K. Anderson, R. Jenness, and W. W. Spink. 1965. Significance of $7 \mathrm{~S}$ and macroglobulin brucella agglutinins in human brucellosis. N. Engl. J. Med. 272:1263-1267.
14. Rose, J. E., and M. H. Roepke. 1957. An acidified antigen for detection of nonspecific reactions in the plate-agglutination test for bovine brucellosis. Am. J. Vet. Res. 18:550-555.

15. U.S. Department of Agriculture. 1967. National Animal Diseases Laboratory diagnostic reagents manual 65E, p. 10. U.S. Department of Agriculture, Animal Health Division, Ames, Iowa. 